\title{
PUDRICIÓN BASAL CAUSADA POR Phytophthora capsici EN PLANTAS DE CHILE TRATADAS CON VERMICOMPOST ${ }^{1}$
}

\author{
Lidieth Uribe-Lorín ${ }^{2}$, Leida Castro-Barquero ${ }^{2}$, Felipe Arauz-Cavallini ${ }^{3}$, Carlos Henríquez-Henríquez ${ }^{2}$, \\ Mónica Blanco-Meneses ${ }^{3}$
}

\begin{abstract}
RESUMEN
Pudrición basal causada por Phytophthora capcisi en plantas de chile tratadas con vermicompost. El objetivo de este trabajo fue evaluar a nivel de invernadero el efecto de vermicompost sobre la incidencia y severidad de Phytophthora capcisi en plantas de chile (Capsicum annuum). Esta investigación se realizó entre febrero y marzo del 2012 en el Centro de Investigaciones Agronómicas, Sabanilla, San José, Costa Rica. Se utilizó un diseño completamente al azar con cuatro repeticiones y ocho plantas por unidad experimental en un arreglo factorial con los factores de dosis $(0,25 \%$ y $50 \%$ v/v vermicompost:suelo) e inoculación (0 y 500 zoosporas por gramo de suelo). Se trasplantaron plántulas de 35 días, que fueron inoculadas dos semanas después del trasplante. La aplicación de vermicompost provocó un aumento significativo del peso fresco foliar y del peso seco foliar y radical; a mayor dosis, mayor fue el incremento. Las plantas de todos los tratamientos que incluyeron inoculación con $P$. capsici presentaron síntomas de la enfermedad en la raíz. Los valores de incidencia y severidad fueron mayores para el tratamiento con $50 \%$ de abono. En este tratamiento se presentaron síntomas de marchitez. La ausencia de diferencias en las variables de peso fresco y seco entre los tratamientos inoculados y sin inocular, a los que se adicionó abono al $25 \%$, sugiere que esta dosis podría compensar el daño causado por el patógeno. Se observó una menor concentración de nutrimentos en los tejidos de las plantas inoculadas lo que indica que el daño causado a la raíz pudo haber afectado la adquisición de nutrimentos.
\end{abstract}

Palabras clave: Capsicum annuum, supresión de enfermedades, pudrición basal del tallo, enmiendas orgánicas.

\begin{abstract}
Effect of vermicompost on pepper plants inoculated with Phytophthora capcisi. The objective of this paper was to evaluate under greenhouse conditions the effect of the application of vermicompost on incidence and severity of Phytophthora capcisi on pepper plants (Capsicum annuum). The experiment was conducted in February and March, 2012, at the Centro de Investigaciones Agronómicas, Sabanilla, San José, Costa Rica. A completely randomized design with 4 replicates and 8 plants per experimental unit, in a factorial design with doses $(0,25 \%$ and $50 \% \mathrm{v} / \mathrm{v}$ compost: soil), and inoculation ( 0 and 500 zoospores per gram of soil) was used. After 35 days, seedlings were transplanted, and two weeks later the plants were inoculated. The application of vermicompost caused a significant increase in fresh and dry shoot weight and dry root weigth, the higher the dose, the greater the increase. Plants of all treatments in which $P$. capsici was inoculated showed root symptoms; the treatment with $50 \%$ compost resulted in symptoms of wilt and the highest incidence and severity of the disease. The lack of differences in fresh and dry weight between inoculated and uninoculated treatments with doses of $25 \%$ of compost, suggest that the last could compensate for the damage caused by the disease. An observed lower concentration of nutrients in the plant tissues inoculated with the pathogen indicates that damage to the root may have affected nutrient uptake.
\end{abstract}

Keywords: Capsicum annuum, disease suppression, Phytophtora blight of pepper, organic amendments.

\footnotetext{
1 Recibido: 14 de marzo, 2013. Aceptado: 30 de junio, 2014. Este trabajo fue financiado por los proyectos VI 733-A9-012 y VI 733-A1-825 de la Universidad de Costa Rica. San José, Costa Rica.

2 Centro de Investigaciones Agronómicas, Universidad de Costa Rica. San José, Costa Rica. lidieth.uribe@ucr.ac.cr (Autora para correspondencia), leida.castro@ucr.ac.cr, carlos.henriquez@ucr.ac.cr.

3 Centro de Investigación en Protección de Cultivos.San José, Costa Rica. felipe.arauz@ucr.ac.cr, monica.blancomeneses@ucr.ac.cr
} 


\section{INTRODUCCIÓN}

La producción de chile dulce (Capsicum annuиm L.) tanto en Costa Rica como en otras partes del mundo, está limitada por la enfermedad conocida como pudrición basal del tallo o maya, causada por el oomycete Phytophthora capsici Leonian (Solís, 1989; Corrales et al., 1990; Kim et al., 2008). La enfermedad se presenta en áreas tropicales y subtropicales e infecta tanto raíces, tallos, hojas y frutos (Kim et al., 1997), siendo una de las enfermedades más destructivas a nivel mundial (Ristaino y Johnston, 1999; Hausbeck y Lamour, 2004). El patógeno causa pudrición de las raíces de la planta y lesiones negras en el tallo; circulares, acuosas y de color café grisáceo en las hojas, y cubiertas de esporangios blancos en los frutos. Las lesiones en tallos y hojas son comunes cuando el inóculo es dispersado por salpique del suelo a las partes inferiores de la planta (Ristaino y Johnston, 1999).

Debido a la naturaleza persistente del inóculo en el suelo, la estrategia para el control de este patógeno requiere un programa de manejo integral (Kim et al., 2008). Sin embargo, las medidas de control químico y cultural no siempre logran disminuir el inóculo de P. capsici, formado por zoosporas con alta movilidad en el suelo. Además, los fungicidas y fumigantes presentan un alto costo de aplicación, potencial daño ambiental, toxicidad y generación de resistencia en los cultivos (Cohen y Coffey, 1986; Lamour y Hausbeck, 2000; Lamour y Hausbeck, 2003). La presión continua de la enfermedad y la preocupación por el impacto ambiental causado por aplicaciones excesivas de productos químicos, hacen necesarias estrategias alternativas para el manejo de $P$. capsici (Hoitink et al., 1997; Chae et al., 2006).

La utilización de enmiendas orgánicas ha recibido mucha atención en las décadas recientes ya que mejora las propiedades físicas, químicas y biológicas del suelo y estimula el crecimiento de las plantas (Zebarth et al., 1999; Barzegar et al., 2002). También se ha demostrado que tienen un efecto supresivo sobre enfermedades ocasionadas por patógenos de suelo (Kim et al., 1997; Noble y Coventry, 2005), incluyendo las causadas por Phytophthora spp. (Kim et al., 1997; Aryantha et al., 2000; Chae et al., 2006; Ntougias et al., 2008). Al respecto, Ntougias et al. (2008) al estudiar el uso de nueve tipos de compost sobre Phytophthora nicotianae en tomate, y Szczech y
Smolinska (2011) utilizando vermicompost producido a partir de estiércoles, reportaron altos niveles de supresión del patógeno.

Se han propuesto varios mecanismos para explicar la supresión de enfermedades por el uso de abonos orgánicos, entre ellos: la estimulación de comunidades nativas de antagonistas, la introducción de agentes de biocontrol al suelo, el aporte de nutrientes para el establecimiento y actividad de dichos organismos, la inducción de resistencia, el aumento en la disponibilidad de nutrimentos a la planta y la mejora en la condición de la raíz, todo ello favorece un crecimiento adecuado del cultivo y le permite tolerar las enfermedades o escapar de la infección (Huber, 1980; Huber, 1991; Hoitink y Grebus, 1994; Hointink y Boehm, 1999; Aryantha et al., 2000; Chae et al., 2006; Termorshuizen et al., 2006; Danon et al., 2007; Artavia et al., 2010).

El grado de supresión que se ha observado es muchas veces impredecible y varía de acuerdo a factores como el tipo de biomasa de origen, el proceso de compostaje, la estabilidad del producto, el tipo de aplicación y la dosis empleada (Hoitink et al., 2006; Rijn et al., 2007; Lozano et al., 2009; Artavia et al., 2010). Al respecto, Bonanomi et al. (2010) al analizar 1964 experimentos observaron supresión en el 45\% de los casos, ningún efecto en el $35 \%$ e incremento en la incidencia de la enfermedad en el $20 \%$ restante. En ensayos a nivel de campo realizados en Costa Rica sobre la supresión de P. capcisi con el uso de abonos orgánicos, Corrales et al. (1990) encontraron que la incorporación de 4 t/ha de gallinaza al suelo redujo la incidencia de la enfermedad a un nivel de $29 \%$, mucho menor que los valores presentados por el tratamiento químico (87\%) y el uso de compost a base de bagazo y cachaza (65\%). Las plantas a las que se les aplicó gallinaza presentaron mayor área foliar, floración y rendimiento que el testigo químico. Hidalgo (2009) a su vez, encontró que la adición de vermicompost a base de estiércol favoreció el desarrollo de la enfermedad, aunque se observó un incremento en las variables de desarrollo de la planta con el uso del abono.

La presente investigación tuvo como objetivo evaluar el efecto de la aplicación de un vermicompost de origen bovino sobre el crecimiento del chile dulce y sobre la incidencia y severidad de los daños causados por $P$. capsici. 


\section{MATERIALES Y MÉTODOS}

\section{Aislamiento e identificación de Phytophtora capcisi.}

El aislamiento de P. capcisi se obtuvo a partir de plantas de chile colectadas en una finca en el Guarco de Cartago que mostraban síntomas de la enfermedad, las plantas se trasladaron al Laboratorio de Microbiología Agrícola del Centro de Investigaciones Agronómicas donde se realizó el aislamiento del oomycete. Se hicieron cortes a partir de zonas del tallo que presentaban lesiones, las secciones se desinfectaron con cloro, se incubaron en placas de petri con $10 \mathrm{ml} \mathrm{de}$ agua desionizada estéril hasta que se desarrolló micelio y se colocaron sobre placas petri con medio PDA para el crecimiento del oomycete. La cepa se identificó por sus características culturales y morfológicas como Phytophthora sp. (Figura 1).

La identificación molecular se realizó en el Laboratorio de Técnicas Moleculares aplicadas a la Fitoprotección del Centro de Investigación en Protección de Cultivos (CIPROC), para lo cual se extrajo ADN a partir de micelio fresco de la cepa, utilizando el método CTAB (Trout et al., 1997). Se realizó la reacción en cadena de la polimerasa (PCR) con los cebadores específicos ITS4 e ITS5, que cubren la secuencia parcial del ARN del gen ribosomal 18S, el espaciador interno transcrito 1, el ARN del gen ribosomal 5.8S, el espaciador interno transcrito 2 y la secuencia parcial del ARN del gen ribosomal 28S. La presencia de una sola banda fue confirmada mediante electroforesis en gel de agarosa $(0,8 \%)$. La secuenciación se realizó al producto purificado de PCR a una concentración de $50 \eta \mathrm{g} / \mu 1$, en la empresa Macrogen Inc. utilizando un secuenciador de Applied Biosystems 3730XL. Los resultados según el BLAST (GenBank-NCBI) muestran que la secuencia en un total de 826 nucleótidos analizados tiene una similitud de $100 \%$ con aislamientos de Phytophthora capcisi (Acc. No GU111643.1, entre otras). La secuencia fue ingresada en GenBank bajo el número de accesión KC677731.

\section{Ensayo de invernadero}

El ensayo se realizó en los meses de febrero y marzo del 2012 en los invernaderos del Centro de Investigaciones Agronómicas. Se utilizó suelo proveniente de Chachagua, San Carlos, Alajuela. El suelo se trató con vapor a $96{ }^{\circ} \mathrm{C}$ por 24 horas. Posteriormente se mezcló con vermicompost de
$\mathbf{A}$

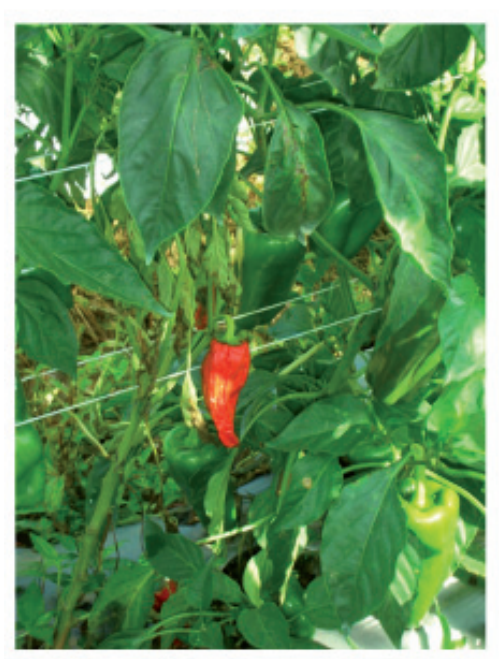

B

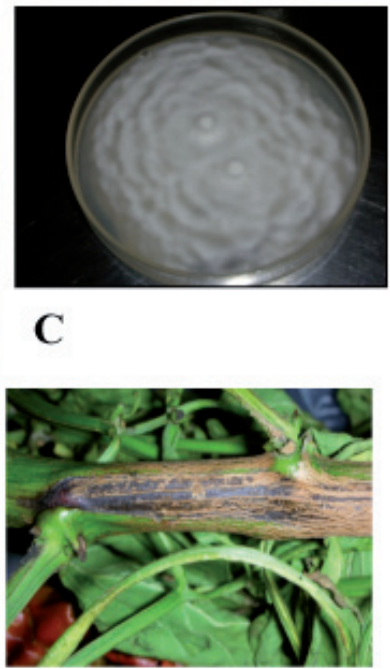

D

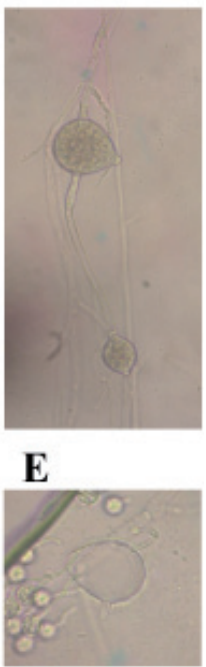

Figura 1. Aislamiento de Phytophthora capcisi a partir de plantas con síntomas en una plantación de chile. [A]: Planta con síntomas de marchitez. [B]: Crecimiento de P. capcisi en PDA. [C]: Tallo sintomático [D]: Esporangios. [E]: Zoosporas. Guarco, Cartago, Costa Rica. Junio, 2010. 
origen bovino procedente de la Sede del Atlántico, Recinto Turrialba, en una proporción de $0 \%, 25 \%$ y $50 \%$ v/v abono:suelo, se colocó en macetas y se incubó durante cinco semanas. Tanto el suelo como el abono se analizaron en el Laboratorio de Suelos y Foliares del Centro de Investigaciones Agronómicas de la Universidad de Costa Rica. Se utilizó un diseño experimental irrestricto al azar con cuatro repeticiones, cada unidad experimental consistió en ocho plantas de chile que fueron trasplantadas a los 35 días de edad en macetas de $200 \mathrm{ml}$ de capacidad. La inoculación se realizó a los catorce días después del trasplante (ddt). El riego se aplicó manteniendo una lámina de $4 \mathrm{~cm}$ de agua en el recipiente en el que se colocó la maceta. Los datos se analizaron con el programa INFOSTAT, mediante un análisis de varianza (ANDEVA) con un arreglo factorial. Los factores analizados fueron: dosis (0, 25\% y $50 \%$ v/v vermicompost:suelo), e inoculación (0 y 500 zoosporas por gramo de suelo). Para la separación de medias se utilizó LSD Fisher $(\mathrm{p} \leq 0,05)$.

\section{Preparación del inoculo de $P$. capcisi}

Para la preparación del inóculo se utilizaron las metodologías descritas por Kim et al. (1997) y Fernández et al. (2007) de la siguiente manera: a partir de un cultivo de P. capcisi de diez días de crecimiento en medio de PDA, se cortaron diez secciones de 5 $\mathrm{mm}$ de diámetro del medio conteniendo micelio. Los discos se colocaron en placas de petri con $20 \mathrm{ml} \mathrm{de}$ agua desionizada estéril. El material se incubó a $25^{\circ} \mathrm{C}$ durante quince días para la formación de esporangios, la liberación de zoosporas se logró al enfriar los cultivos a $5{ }^{\circ} \mathrm{C}$ por una hora, seguida por incubación a $25{ }^{\circ} \mathrm{C}$ por 30 minutos. La concentración de zoosporas fue determinada usando un hemacitómetro después de agitar por un minuto con un vortex a fin de inducir las zoosporas a enquistarse (Kim et al., 1997). La suspensión se diluyó con agua desionizada estéril para obtener un inóculo de $6 \times 10^{4}$ zoosporas/ml (aprox. 500 zoosporas/g de suelo). Cada planta se inoculó con $1 \mathrm{ml}$ de la suspensión de zoosporas. Las plantas del control se trataron con agua desinoizada estéril.

\section{Variables evaluadas}

Se determinó el peso fresco de follaje y raíz, así como el peso después de secar el material a $60{ }^{\circ} \mathrm{C}$ por dos días. Además, se evaluó la incidencia y severidad de la enfermedad tanto para la parte radical como para la parte aérea utilizando como base las escalas de síntomas indicadas en las Figuras 2 y 3.
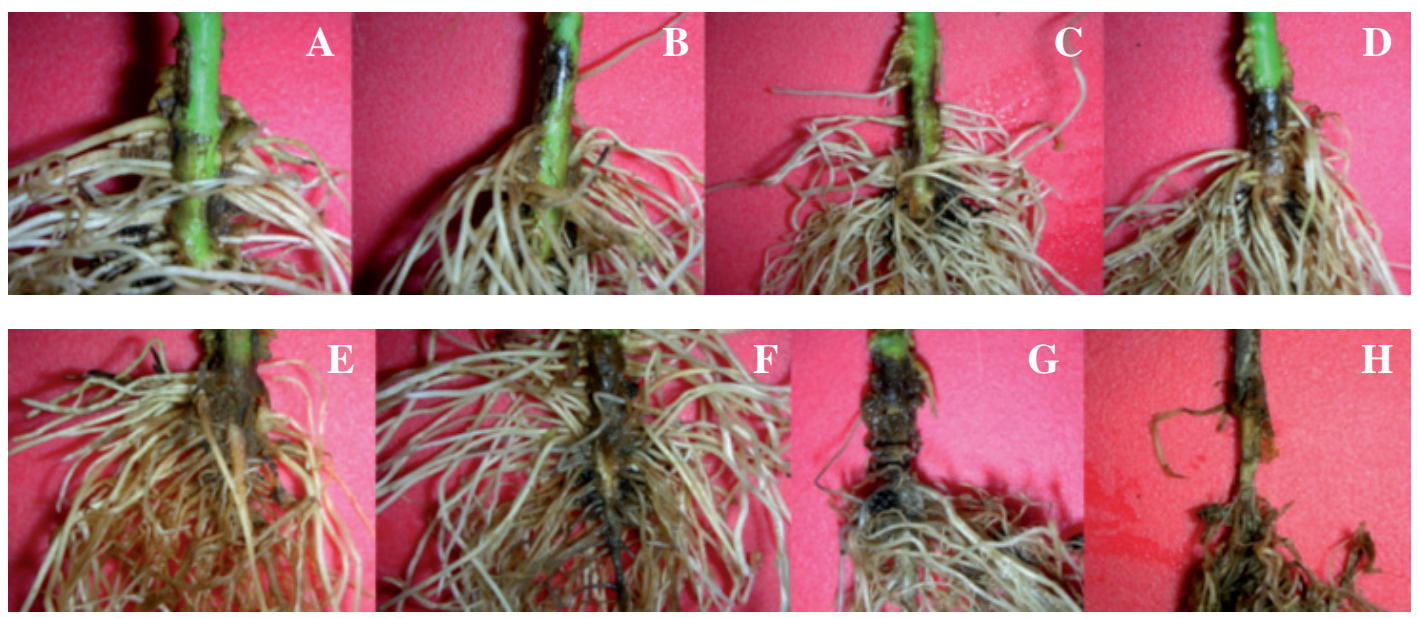

Figura 2. Escala de severidad del ataque de P. capcisi en la raíz de las plantas de chile Capsicum annuum var Natalie. [A]: Lesión circular seca [B]: Necrosis ligera en la base de la raíz, lesión mayor de 0,5 cm. [C]: Necrosis seca aprox $1,5 \mathrm{~cm}$, suberizadas, raíces adventicias [D]: Raíz que presenta lesiones con necrosis de $2 \mathrm{~cm}$ [E]: Lesiones con necrosis de $2 \mathrm{~cm}$, raíces empiezan a desprenderse [F]: Lesiones con necrosis de $2 \mathrm{~cm}$, se desprende gran cantidad de raíces [G]: Se desprende epidermis dejando tejido vascular, pérdida de $50 \%$ de raíces y $[\mathrm{H}]$ : Desprendimiento $80 \%$ raíces se expone tejido pérdida de epidermis, raíces necróticas. Centro de Investigaciones Agronómicas, Universidad de Costa Rica, Sabanilla, Costa Rica. Marzo, 2012. 


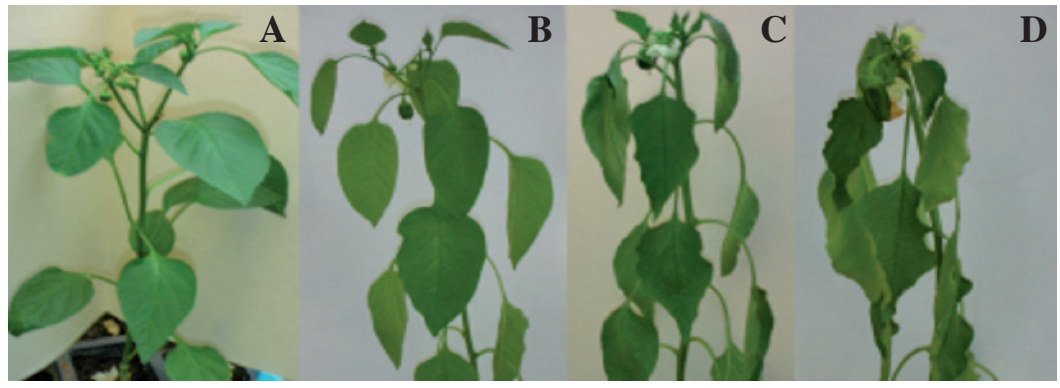

Figura 3. Escala de severidad del ataque de P. capcisi de la parte aérea de las plantas de chile Capsicum annuum var Natalie. [A]: Planta sana. [B]: Planta con algunas hojas con pérdida de turgencia. [C]: Plantas con todas las hojas con pérdida de turgencia [D]: Plantas con hojas marchitas. Centro de Investigaciones Agronómicas. Universidad de Costa Rica, Sabanilla, Costa Rica. Marzo, 2012.

\section{RESULTADOS Y DISCUSIÓN}

El suelo utilizado como sustrato tiene una fertilidad de media a baja y a pesar de los bajos contenidos de $\mathrm{Mg}$ y $\mathrm{K}$ mostrados, no manifestó problemas de acidez. El nivel de $\mathrm{P}$ disponible estuvo por debajo de los límites detectables en el análisis de suelos y el contenido de materia orgánica fue levemente bajo para este tipo de suelo (Cuadro 1).

Elvermicompost mostró niveles comparativamente altos de elementos con respecto a otros abonos orgánicos (Durán y Henríquez, 2007). El pH obtenido $(6,7)$, evidencia el alto grado de estabilidad del material. Szczech y Smolinska (2011), indican que abonos a base de estiércoles tienen $\mathrm{pH}$ cercano a la neutralidad o ligeramente alcalinos y un mayor contenido de nutrimentos comparados con los de origen vegetal (Castro et al., 2009), por lo que su uso favorece las propiedades físicas, químicas y microbiológicas del suelo y el desarrollo de la planta. La relación $\mathrm{C} / \mathrm{N}$ fue de 10,5, en este sentido Day y Shaw (2001), indican que un valor de $\mathrm{C} / \mathrm{N}$ entre 10 y 20 es aceptable para abonos orgánicos, los materiales con relaciones $\mathrm{C} / \mathrm{N}$ $<10$ permitan una mayor liberación de nutrimentos que aquellos con valores >20 (Fassbender y Bornemisza, 1987; Day y Shaw, 2001) (Cuadro 2).

El alto valor de conductividad eléctrica presentado $(19,7 \mathrm{mS} / \mathrm{cm})$ se atribuye a que el proceso de vermicompostaje se realizó bajo techo, lo que reduce la lixiviación de nutrientes y permite una mayor concentración de los mismos en forma de sales (Vandergheynst et al., 2004).

Las sales presentes en los abonos orgánicos provienen de los estiércoles, agua, fertilizantes y otros ingredientes utilizados para enmendar el producto final; cuando estas sales se acumulan cerca de la zona radical a un nivel en el cual la raíz no puede extraer suficiente agua, puede reducirse el rendimiento del cultivo (Vandergheynst et al., 2004). Debido a esto, se recomienda que la conductividad eléctrica en abonos utilizados en viveros e invernaderos estén en un rango de 2,0 a 5,0 mS/cm, de manera que cuando el abono se incorpore al medio de crecimiento, la mezcla no exceda $3,0 \mathrm{mS} / \mathrm{cm}$ para viveros y $2,0 \mathrm{mS} / \mathrm{cm}$ para cultivos en invernadero (Miller, 2001; Dimambro et al., 2007).

A pesar del alto valor de salinidad del vermicompost utilizado, su aplicación a las plantas de chile resultó en

Cuadro 1. Análisis químico del suelo. Chachagua, San Carlos, Alajuela, Costa Rica. 2012.

\begin{tabular}{ccccccccccccc}
\hline $\mathbf{p H}$ & \multicolumn{3}{c}{$\mathbf{c m o l}(+) / \mathbf{l}$} & & \multicolumn{3}{c}{$\mathbf{m g} / \mathbf{l}$} & \multicolumn{3}{c}{$\%$} \\
\hline $\mathbf{H}_{2} \mathbf{O}$ & Acidez & $\mathbf{C a}$ & $\mathbf{M g}$ & $\mathbf{K}$ & $\mathbf{C I C E}$ & $\mathbf{S A}$ & $\mathbf{P}$ & $\mathbf{Z n}$ & $\mathbf{C u}$ & $\mathbf{F e}$ & $\mathbf{M n}$ & $\mathbf{M O}$ \\
\hline 6,5 & 0,15 & 4,53 & 0,89 & 0,01 & 5,58 & 3 & $\mathrm{ND}$ & 1,6 & 8 & 54 & 14 & 1,9 \\
\hline
\end{tabular}


Cuadro 2. Análisis químico del abono orgánico procedente de la Sede de Turrialba, Cartago, Costa Rica. 2012.

\begin{tabular}{ccccccccccccc}
\hline $\mathbf{p H}$ & \multicolumn{1}{c}{} & \multicolumn{3}{c}{$\%$} & \multicolumn{1}{c}{$\mathbf{m g} / \mathbf{k g}$} & \multicolumn{3}{c}{$\mathbf{m S} / \mathbf{c m}$} \\
\hline $\mathbf{H}_{2} \mathbf{O}$ & $\mathbf{H u m}$ & $\mathbf{P}$ & $\mathbf{C a}$ & $\mathbf{M g}$ & $\mathbf{K}$ & $\mathbf{S}$ & $\mathbf{F e}$ & $\mathbf{C u}$ & $\mathbf{Z n}$ & $\mathbf{M n}$ & $\mathbf{B}$ & $\mathbf{C E}$ \\
\hline 6,7 & 53 & 2,98 & 5,25 & 1,03 & 1,04 & 0,68 & 4637 & 238 & 940 & 637 & 23 & 19,7 \\
\hline
\end{tabular}

un aumento significativo de su crecimiento evaluado como peso fresco y seco foliar, y peso seco radical, a mayor dosis, mayor el incremento (Cuadro $3 \mathrm{y}$ Figura 4). Resultados similares fueron encontrados por Sanchez-Monedero et al. (2004) al evaluar once sustratos que presentaban valores de salinidad por encima de los niveles de referencia para su utilización en cultivos (3,2 a 13,2 mS/cm). Estos sustratos, a pesar de la alta salinidad, tuvieron efectos beneficiosos sobre el crecimiento de plantas de brócoli, tomate y cebolla en condiciones de viveros comerciales. Un efecto positivo en el crecimiento y producción de plantas de chile, fue también encontrado por Arancon et al. (2005) al utilizar vermicompost de diferentes fuentes, los autores atribuyeron el aumento en el área foliar y en la biomasa de la parte aérea, a los nutrimentos, materia orgánica, hormonas u otros reguladores de crecimiento aportados por los abonos.

$\mathrm{La}$ incorporación de nutrimentos por medio de la adición del vermicompost se muestra en el Cuadro 4. Las plantas presentaron un mayor contenido de $\mathrm{N}$, $\mathrm{P}, \mathrm{Mg}, \mathrm{K}$ y $\mathrm{Cu}$, que las plantas sin abono. Sanchez-
Monedero (2004) también encontró un mayor contenido de elementos en el tejido de plantas que crecieron en sustratos a los que se les adicionó compost.

Cuando las plantas de chile fueron inoculadas con $P$. capcisi, no se observó diferencias en las variables de crecimiento con respecto al tratamiento testigo ( $\sin$ inocular y sin abono). La adición de vermicompost al 25\% aumentó el peso fresco y seco aéreo; sin embargo, una dosis mayor no produjo un aumento en el crecimiento de la planta, por el contrario, el peso fresco radical del tratamiento con $50 \%$ de vermicompost fue significativamente menor que el del tratamiento testigo (Cuadro 3 y Figura 4). Este comportamiento se debe probablemente al daño que causó $P$. capsici en las raíces (Figura 2), el cual afectó la nutrición de las plantas, observándose una menor concentración de nutrimentos en los tejidos del chile inoculado con el patógeno (Cuadro 5).

En el caso del $\mathrm{Fe}$ hay que destacar que al comparar los tratamientos sin adición de abono, el inoculado con P. capcisi (SP), presentó un contenido foliar significativamente menor de este elemento, lo

Cuadro 3. Efecto de la aplicación de vermicompost sobre plantas de chile Capsicum annuum var Natalie inoculadas con Phytophthora capcisi. Centro de Investigaciones Agronómicas, Universidad de Costa Rica, San José, Costa Rica. Marzo, 2012.

\begin{tabular}{lcccc}
\hline Tratamiento & Peso fresco aéreo $(\mathbf{g})$ & Peso fresco radical $(\mathbf{g})$ & Peso seco aéreo $(\mathbf{g})$ & Peso seco radical $(\mathbf{g})$ \\
\hline $\mathrm{S}$ & $46,57 \mathrm{a}$ & $39,21 \mathrm{bcd}$ & $7,33 \mathrm{a}$ & $4,01 \mathrm{a}$ \\
$\mathrm{SV} 25 \%$ & $97,94 \mathrm{~b}$ & $42,81 \mathrm{~cd}$ & $12,90 \mathrm{~b}$ & $4,09 \mathrm{a}$ \\
$\mathrm{SV} 50 \%$ & $116,57 \mathrm{c}$ & $44,15 \mathrm{~d}$ & $17,13 \mathrm{c}$ & $4,83 \mathrm{~b}$ \\
$\mathrm{SP}$ & $42,10 \mathrm{a}$ & $33,08 \mathrm{ab}$ & $6,83 \mathrm{a}$ & $3,60 \mathrm{a}$ \\
$\mathrm{SV} 25 \% \mathrm{P}$ & $87,65 \mathrm{~b}$ & $35,00 \mathrm{abc}$ & $13,18 \mathrm{~b}$ & $3,54 \mathrm{a}$ \\
SV50\% P & $83,45 \mathrm{~b}$ & $29,22 \mathrm{a}$ & $12,55 \mathrm{~b}$ & $3,44 \mathrm{a}$ \\
\hline $\mathrm{p}$ & $<0,0001$ & 0,0174 & $<0,0001$ & 0,01 \\
\hline
\end{tabular}

(S) Suelo (SV25\%) Suelo + vermicompost al 25\%, (SV50\%) Suelo + vermicompost al 50\%, (SP) Suelo + P. capcisi, (SV25\%P) Suelo + vermicompost al 25\% + P. capcisi, (SV50\%P) Suelo + vermicompost al 50\% + P. capcisi.

Letras diferentes en la misma columna implican diferencias significativas según LSD p<0,05). 


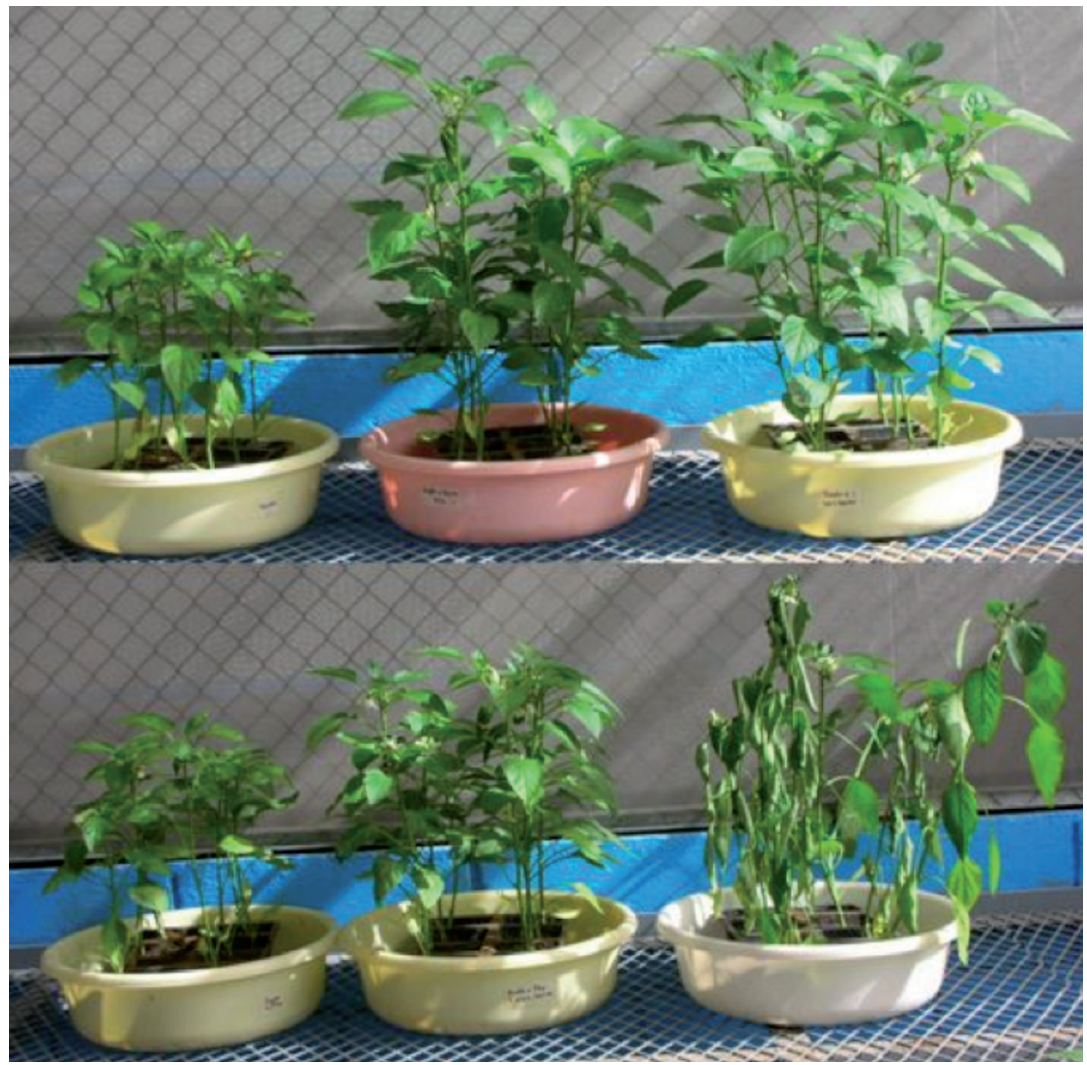

Figura 4. Efecto de la adición de vermicompost y la inoculación con P. capcisi sobre plantas de chile Capsicum annuиm var Natalie. [A]: Suelo, [B]: Suelo + vermicompost al 25\%, [C]: Suelo + vermicompost al 50\%, [D]: Suelo + P. capcisi, $[\mathrm{E}]:$ Suelo + vermicompost al 25\% + P. capcisi, $[\mathrm{F}]$ : Suelo + vermicompost al $50 \%+P$. capcisi. Centro de Investigaciones Agronómicas, Universidad de Costa Rica, San José, Costa Rica. Marzo, 2012.

Cuadro 4. Contenido nutricional de las plantas de chile Capsicum annuum var Natalie según la dosis de vermicompost aplicada. Centro de Investigaciones Agronómicas, Universidad de Costa Rica, San José, Costa Rica. Marzo, 2012.

\begin{tabular}{lcccccccc}
\hline \multirow{2}{*}{$\begin{array}{l}\text { Dosis } \\
\text { de abono }\end{array}$} & $\mathbf{N}$ & $\mathbf{P}$ & $\mathbf{C a}$ & $\mathbf{M g}$ & $\mathbf{K}$ & $\mathbf{S}$ & $\begin{array}{c}\mathbf{C u} \\
\mathbf{M g} / \mathbf{k g}\end{array}$ \\
\cline { 2 - 9 } $0 \%$ & $2,2 \mathrm{a}$ & $0,31 \mathrm{a}$ & $1,84 \mathrm{a}$ & $0,50 \mathrm{a}$ & $2,18 \mathrm{a}$ & $0,40 \mathrm{a}$ & $7,25 \mathrm{a}$ \\
$25 \%$ & $2,47 \mathrm{~b}$ & $0,34 \mathrm{~b}$ & $1,79 \mathrm{a}$ & $0,69 \mathrm{~b}$ & $3,84 \mathrm{~b}$ & $0,38 \mathrm{a}$ & $8,38 \mathrm{~b}$ \\
$50 \%$ & $3,05 \mathrm{c}$ & $0,36 \mathrm{~b}$ & $1,74 \mathrm{a}$ & $0,76 \mathrm{c}$ & $5,12 \mathrm{c}$ & $0,40 \mathrm{a}$ & $8,38 \mathrm{~b}$ \\
\hline $\mathrm{p}$ & $<0,001$ & 0,0008 & 0,3504 & $<0,0001$ & $<0,0001$ & 0,1176 & 0,0493 \\
\hline
\end{tabular}

Letras diferentes en la misma columna implican diferencias significativas según LSD p<0,05). 
Cuadro 5. Contenido de nutrientes en los tejidos de las plantas de chile Capsicum annuum var Natalie inoculadas y no inoculadas con Phytophthora capsici. Centro de Investigaciones Agronómicas, Universidad de Costa Rica, San José, Costa Rica. Marzo, 2012.

\begin{tabular}{|c|c|c|c|c|c|c|c|}
\hline \multirow[t]{2}{*}{ Tratamiento } & $\mathbf{N}$ & $\mathbf{P}$ & $\mathbf{C a}$ & $\mathrm{Mg}$ & $\mathbf{K}$ & $\mathbf{S}$ & \multirow{2}{*}{$\begin{array}{c}\mathrm{Cu} \\
(\mathrm{mg} / \mathrm{kg}) \\
\end{array}$} \\
\hline & & & $\%$ & & & & \\
\hline Sin inocular & $2,64 \mathrm{a}$ & $0,35 \mathrm{~b}$ & $1,90 \mathrm{~b}$ & $0,69 \mathrm{~b}$ & $3,94 \mathrm{~b}$ & $0,41 \mathrm{~b}$ & $9,0 \mathrm{~b}$ \\
\hline Inoculado & $2,50 \mathrm{a}$ & $0,32 \mathrm{a}$ & $1,67 \mathrm{a}$ & $0,61 \mathrm{a}$ & $3,49 \mathrm{a}$ & $0,37 \mathrm{a}$ & $7,0 \mathrm{a}$ \\
\hline $\mathrm{p}$ & 0,0788 & 0,0009 & 0,0005 & 0,0014 & 0,001 & $<0,0001$ & 0,0001 \\
\hline
\end{tabular}

Letras diferentes en la misma columna implican diferencias significativas según LSD $p<0,05)$.

que parece indicar algún tipo de competencia por el hierro en el sistema planta-patógeno, mientras que en los tratamientos con vermicompost y $P$. capcisi, los contenidos de Fe no difirieron significativamente del tratamiento control, al parecer la adición del abono aporta al sistema el Fe necesario (Cuadro 6).

Todos los tratamientos inoculados con P. capsici mostraron síntomas de la enfermedad en la parte radical (Cuadro 7, Figura 2), siendo los valores de incidencia y severidad mayores para el tratamiento con 50\% de vermicompost que presentó además marchitez (Figura 3D). Estos resultados coinciden

Cuadro 6. Efecto de la inoculación con P. capcisi y la adición de vermicompost sobre el contenido de Fe en los tejidos de plantas de $C$. annuun. Centro de Investigaciones Agronómicas, Universidad de Costa Rica, San José, Costa Rica. Marzo, 2012.

\begin{tabular}{lc}
\hline Tratamientos & $\begin{array}{c}\text { Fe } \\
(\mathbf{m g} / \mathbf{k g})\end{array}$ \\
\hline $\mathrm{S}$ & $156,75 \mathrm{~b}$ \\
$\mathrm{SV} 25 \%$ & $136,50 \mathrm{ab}$ \\
$\mathrm{SV} 50 \%$ & $149,25 \mathrm{~b}$ \\
$\mathrm{SP}$ & $96,50 \mathrm{a}$ \\
$\mathrm{SV} 25 \% \mathrm{P}$ & $127,25 \mathrm{ab}$ \\
$\mathrm{SV} 50 \% \mathrm{P}$ & $172,25 \mathrm{~b}$ \\
\hline $\mathrm{p}$ & 0,0476 \\
\hline
\end{tabular}

(S) Suelo (SV25\%) Suelo + vermicompost al 25\%, (SV50\%) Suelo + vermicompost al $50 \%$, (SP) Suelo + P. capcisi, (SV25\%) Suelo + vermicompost al $25 \%+$ P. capcisi, (SV50\%P) Suelo + vermicompost al 50\% + P. capcisi.

Letras diferentes en la misma columna implican diferencias significativas según LSD $\mathrm{p}<0,05$ ). con lo observado por Kim et al. (1997) quienes señalan que el uso de humatos y lodos composteados incrementaron significativamente el daño causado por Phytophthora en la raíz y en la corona del tallo de plantas de chile, además redujeron el peso seco de raíces y tallos, y con Hidalgo (2009) al utilizar a nivel de campo, el vermicompost proveniente de la Sede de Turrialba. Contrariamente, Corrales et al. (1990) encontraron a nivel de campo en Costa Rica, que el uso de gallinaza redujo significativamente la incidencia de la enfermedad. Además, Aryantha et al. (2000) observaron también que el uso de gallinaza fresca y composteada redujo la sobrevivencia de $P$. cinnamomi y el desarrollo de la enfermedad en plantas de Lupinus albus, mientras que la adición de estiércol de vacuno, ovino y equino, tanto fresco como composteado no logró suprimir las poblaciones del patógeno ni los síntomas de la enfermedad. Estos resultados indican que el origen y proceso del material utilizado son factores importantes en la supresión de las enfermedades y que mayor investigación debe realizarse en este tema, especialmente si se considera que los residuos orgánicos son frecuentemente inefectivos o conductivos para patógenos con una alta capacidad saprofítica (Hoitink et al., 1997; Bonanomi et al., 2010). Al analizar el resultado de 28 experimentos en los cuales se evaluó el efecto de enmiendas orgánicas sobre Phytophthora spp., Bonanomi et al. (2010) encontraron que en un 50\% de los casos no se encontró ninguna respuesta, en el $25 \%$ fue positiva (reducción de las poblaciones del patógeno) mientras que en el otro $25 \%$ se incrementó el desarrollo de las poblaciones del patógeno y la incidencia de la enfermedad.

$\mathrm{Si}$ bien la enfermedad fue más severa en el tratamiento suelo con vermicompost al $25 \%$ e 
Cuadro 7. Efecto de la aplicación de vermicompost sobre plantas de chile inoculadas con Phytophthora sp. Centro de Investigaciones Agronómicas, Universidad de Costa Rica, San José, Costa Rica. Marzo, 2012.

\begin{tabular}{lccccc}
\hline \multirow{2}{*}{ Tratamiento } & \multicolumn{2}{c}{ Raíces } & & \multicolumn{2}{c}{ Parte aérea } \\
\cline { 2 - 3 } \cline { 5 - 6 } \cline { 5 - 6 } & Incidencia \% & Severidad & & Incidencia \% & Severidad \\
\hline SV25\% & 0 & 0 & & 0 & 0 \\
SV50\% & 0 & 0 & & 0 & 0 \\
SP & 0 & $0,8 \mathrm{a}$ & & 0 & 0 \\
SV25\%P & $65,6 \mathrm{a}$ & $2,9 \mathrm{~b}$ & & 0 & 0 \\
$\mathrm{SV} 50 \% \mathrm{P}$ & $71,9 \mathrm{ab}$ & $6,1 \mathrm{c}$ & & 46,9 & 1,3 \\
\hline $\mathrm{p}$ & $96,9 \mathrm{~b}$ & $<0,0001$ & & & \\
\hline
\end{tabular}

(S) Suelo (SV25\%) Suelo + vermicompost al 25\%, (SV50\%) Suelo + vermicompost al 50\%, (SP) Suelo + P. capcisi, (SV25\%P) Suelo + vermicompost al 25\% + P. capcisi, (SV50\%P) Suelo + vermicompost al $50 \%+$ P. capcisi.

Letras diferentes en la misma columna implican diferencias significativas según LSD p<0,05).

inoculado con Phytophthora (SV25\%P) que en el inoculado con el patógeno (SP), no se observaron diferencias en las variables de peso fresco y seco entre estos tratamientos, al parecer con esta dosis de abono, la planta compensa el efecto de la enfermedad.

El abono afectó negativamente solo a las plantas inoculadas con Phytophthora, lo que podría atribuirse a que los compost salinos pueden aumentar las enfermedades causadas por Pythium y Phytophthora, a menos que se apliquen meses antes de la siembra (Hoitink et al., 1997). Al respecto, Sanogo (2004) encontró que la severidad de los síntomas causados por P. capsici en plantas de chile aumentó con la salinidad del sustrato, debido a efectos directos e indirectos sobre el patógeno, la planta y su interacción. En ese estudio la alta salinidad causó daño directo a la planta y un aumento en el crecimiento micelial de $P$. capsici, y al mismo tiempo una disminución en la producción de esporangios y zoósporas. Una mayor salinidad en la dosis más alta, probablemente explica el efecto encontrado respecto a la enfermedad en las plantas a las que se adicionó vermicompost al 50\%.

\section{LITERATURA CITADA}

Arancon, N., C. Edwards, P. Biermanb, J. Metzgerc, y C. Luchtd. 2005. Effects of vermicomposts produced from cattle manure, food waste and paper waste on the growth and yield of peppers in the field. Pedobiologia 49(4):297-306.
Artavia, S., L. Uribe, F. Saborío, L.F. Arauz, y L. Castro. 2010. Efecto de la aplicación de abonos orgánicos en la supresión de Pythium myriotylum en plantas de tiquisque (Xanthosoma sagittifolium). Agron. Costarricense 34(1):17-29.

Aryantha, I.P., R. Cross, y D.I. Guest. 2000. Suppression of Phytophthora cinnamomi in potting mixes amended with uncomposted and composted animal manures. Phytopathology 90:775-782.

Barzegar, A.R., A. Yousefi, y A. Daryashenas. 2002. The effect of addition of different amounts and types of organic materials on soil physical properties and yield of wheat. Plant Soil 247:295-301.

Bertsch, F. 1995. Fertilidad de suelos y su manejo. Asociación Costarricense de la Ciencia del Suelos (ACCS), San José, Costa Rica.

Bonanomi, G., V. Antignani, M. Capodilupo, y F. Scala. 2010. Identifying the characteristics of organic soil amendments that suppress soilborne plant diseases. Soil Biol. Biochem. 42:136-144.

Castro, A., C. Henríquez, y F. Bertsch. 2009. Capacidad de suministro de $\mathrm{N}, \mathrm{P}$ y $\mathrm{K}$ de cuatro abonos orgánicos. Agron. Costarricense 33(1):31-43.

Cohen, Y., y M.D. Coffey. 1986. Systemic fungicides and the control of oomycetes. Annu. Rev. Phytopathol. 24:311-338.

Corrales, O., E. Vargas, y M.A. Moreira. 1990. Efecto de la materia orgánica en el combate de la pudrición basal del chile dulce (Capsicum annuum) causada por Phytophthora capcisi. Agron. Costarricense 14(1):914. 
Chae, D.H., R. Jin, H. Hwangbo, Y.M. Kim, Y.C. Kim, R.D. Park, H.B. Krishnan, y K.Y. Kim. 2006. Control of late blight (Phytophthora capsici) in pepper plant with a compost containing multitude of chitinaseproducing bacteria. BioControl 51:339-351.

Danon, M., S. Zmora-Nahum, Y. Chen, y Y. Hadar 2007. Prolonged compost curing reduces suppression of Sclerotium rolfsii. Soil Biol. Biochem. 39:1936-1946.

Day, M., y K. Shaw. 2001. Biological, chemical and physical processes of composting, En: P. Stofella, y B. Kahn, editores, Compost utilization in horticultural cropping systems. Lewis, USA. p. 18-22.

Dimambro, M.E., R.D. Lillywhite, y C.R. Rahn. 2007. The physical, chemical and microbial characteristics of biodegradable municipal waste derived composts. Compost Sci. Util. 15:243-252.

Durán, L., y C. Henríquez. 2007. Caracterización química, física y microbiológica de vermicompostes producidos a partir de cinco sustratos orgánicos. Agron. Costarricense 31(1):41-51.

Fassbender, H., y E. Bornemisza. 1987. Química de suelos con énfasis en suelos de América Latina. 2 ed. IICA, San José, Costa Rica.

Fernández, E., M. Acosta, y V. Pinto. 2007. Efecto de aplicaciones de fungicidas sobre la incidencia de la marchitez (Phytophthora capsici Leo.) del jitomate (Lycopersicon esculentum Mill.) en invernadero. Rev. Mex. Fitopatol. 25:186-189.

Hausbeck, M.K., y K.H. Lamour. 2004. Phytophthora capsici on vegetable crops: research progress and management challenges. Plant Dis. 88:1292-1303.

Hidalgo, M. 2009. Efecto del vermicompost sobre el cultivo del chile dulce (Capsicum annuum) y su interacción con Phytophthora capsici. Tesis para optar por el título de Licenciatura en Agronomía con énfasis en Fitotecnia, Universidad de Costa Rica, Turrialba, Costa Rica.

Hointink, H.A.J., y M. Boehm. 1999. Biocontrol within the context of soil microbial communities: A substratedependent phenomenon. Annu. Rev. Phytopathol. 37:427-446.

Hoitink, H.A.J., y M. Grebus. 1994. Status of biological control of plant diseases with composts. Compost Sci. Util. 2:6-12.

Hoitink, H.A.J., L.V.. Madden, y A.E. Dorrance. 2006. Systemic resistance induced by Trichoderma spp.: Interactions between the host, the pathogen, the biocontrol agent, and soil organic matter quality. Phytopathology 96:186-189.
Hoitink, H.A.J., A.G. Stone, y D.Y. Han. 1997. Supresión de enfermedades de plantas mediante compost. Agron. Costarricense 21:25-33.

Huber, D.M. 1980. The role of mineral nutrition in disease. En: J.G. Horsfall, y E.B. Cowling, editores, Plant disease, an advanced treatise. Academic Press, NY, USA. p. 381-406.

Huber, D.M. 1991. The use of fertilizers and organic amendments in the control of plant disease. En: D. Pimentel, editor, Handbook of pest management in agriculture. CRC Press, Boca Ratón, FL, USA. p. 405-494.

Kim, H.S., M.K. Sang, Y.C. Jeun, B.K. Hwang, y K.D. Kim. 2008. Sequential selection and efficacy of antagonistic rhizobacteria for controlling Phytophthora blight of pepper. Crop Prot. 27:436-443.

Kim, K.D., S. Nemec, y G. Musson. 1997. Control of Phytophthora root and crown rot of bell pepper with composts and soil amendments in the greenhouse. Appl. Soil Ecol. 5:169-179.

Lamour, K.H., y M.K. Hausbeck. 2000. Mefenoxam insensitivity and the sexual stage of Phytophthora capsici in Michigan Cucurbit Fields. Phytopathology 90:396-400.

Lamour, K.H., y M.K. Hausbeck. 2003. Effect of crop rotation on the survival of Phytophthora capsici in Michigan. Plant Dis. 87:841-845.

Lozano, J., W. Blok, y A. Termorshuizen. 2009. Effect of compost particle size on suppression of plant diseases. Environ. Eng. Sci. 26:601-607.

Miller, M. 2001. Fulfilling special needs of nurseries. BioCycle 42(4):55-59.

Noble, R., y E. Coventry. 2005. Suppression of soil-borne plant diseases with composts: A review. Biocontrol Sci. Techn. 15:3-20.

Ntougias, S., K.K. Papadopoulou, G.I. Zervakis, N. Kavroulakis, y C. Ehaliotis. 2008. Suppression of soil-borne pathogens of tomato by composts derived from agro-industrial wastes abundant in Mediterranean regions. Biol Fertil Soils 44:1081-1090.

Rijn, E., A. Termorshuizen, y A. Van Bruggen. 2007. Storage method affects disease suppression of flax wilt induced by composts. Soil Biol. Biochem. 39: 2743-2749.

Ristaino, J.B., y S.A. Johnston. 1999. Ecologically based approaches to management of Phytophthora blight on bell. Plant Dis. 83:1080-1089.

Sanchez-Monedero, M.A., A. Roig, J. Cegarra, M.P. Bemal, P. Noguera, M. Abad, y A. Antón. 2004. Composts as 
media constituents for vegetable transplant production. Compost Sci. Util. 12:161-168.

Sanogo, S. 2004. Response of chile pepper to Phytophthora capsici in relation to soil salinity. Plant Dis. 88:205209.

Szczech, M., y U. Smolinska. 2011. Comparison of suppressiveness of vermicomposts produced from animal manures and sewage sludge against Phytophthora nicotianae breda de Haan var. nicotianae. J. Phytopathol. 149:77-82.

Solís, V.C. 1989. Índice de enfermedades de los cultivos agrícolas de Costa Rica. Ministerio de Agricultura y Ganadería, San José, Costa Rica.

Termorshuizen, A.J., E. Van Rijn, D.J. Van Der Gaag, C. Alabouvette, Y. Chen, J. Lagerlöf, A.A. Malandrakis, E.J. Paplomatas, B. Rämert, J. Ryckeboer, C.
Steinberg, y S. Zmora-Nahum. 2006. Suppressiveness of 18 composts against 7 pathosystems: Variability in pathogen response. Soil Biol. Biochem. 38:24612477.

Trout,C.L.,J.B.Ristaino,M.Madritch,yT. Wangsomboondee. 1997. Rapid detection of Phytophthora infestans in late blight-infected potato and tomato using PCR. Plant Dis. 81:1042-1048.

Vandergheynst, J.S., S. Pettygrove, T.M. Dooley, y K.A. Arnold. 2004. Estimating electrical conductivity of compost extracts at different extraction ratios. Compost Sci. Util. 12:202-207.

Zebarth, B.J., G.H. Neilsen, E. Hogue, y D. Neilsen. 1999. Influence of organic waste amendments on selected soil physical and chemical properties. Can. J. Soil Sci. 79:501-504. 
